\title{
Social listening during crises: A practitioner guide for crisis communication on social media
}

\author{
Cory Young \\ Ithaca College \\ Ithaca, NY USA \\ Hunter Simmons \\ Ithaca College \\ Ithaca, NY USA \\ Margaret C. Stewart \\ University of North Florida \\ Jacksonville, FL USA
}

\begin{abstract}
Stewart \& Wilson (2016) created the STREMII model (pronounced STREAM-ee) as a means to assist institutions and organizations with social media crisis communication using a six-stage cyclical process, including: (1) social listening, (2) targeting audience(s), (3) engaging \& responding, (4) monitoring and evaluating, (5) interacting, and (6) implementing changes [1]. Stewart \& Young (2017) revisited the model, refining the stages to highlight the need for ongoing social listening and responsive engagement across all levels of crisis [2]. At present, the model is theoretical and applied only within a pedagogical context. In order for the STREMII model to be useful for practical implementation and relevant outside the theoretical and pedagogical contexts, we must develop a practical set of actionable steps for practitioners (crisis communicators and social media strategists). To accomplish this, we surveyed practitioners about the process they engage in listening, interacting and responding to audiences on social media during a crisis, and how they monitor and evaluate their responses and outcomes. The desired outcome of this research is to create a practical set of actionable steps for crisis practitioners and social media strategists, with specific guidelines, considerations and recommendations for adopting the STREMII model and integrating it into existing crisis management plans and social media strategies.
\end{abstract}

Keywords - STREMII Model, Social Media, Crisis Communication, Practitioners

SUGGESTED CITATION: Young, C., Simmons, H., \& Stewart, M. C. (2019). Social listening during crises: A practitioner guide for crisis communication on social media. Proceedings of the International Crisis and Risk Communication Conference, Volume 2 (pp. 38-40). Orlando Fl: Nicholson School of Communication and Media. https://doi.org/10.30658/icrcc.2019.11

\section{INTRODUCTION}

Stewart \& Young (2018) presented research at the International Crisis and Risk Communication annual conference in Orlando, FL that focused on the implementation of the STREMII Model [3] originally developed by Stewart \& Wilson [1]. The proceedings, available through the Nicholson School of Communication and Media at the University of Central Florida, document the recommendations for adopting and integrating the STREMII model into existing crisis management plans and social media strategies. What follows is a brief summary of the suggestions, which span three phases of a crisis: before, during and after.

Before a crisis, it is crucial for organizations to establish a social media strategy to detail how an organization will listen to constituents virtually. Practicing social listening is critical towards identifying and responding to a crisis on social media 
and other digital channels. In addition to listening, it is essential to identify target audiences during the pre-crisis phase, paying careful attention to how the audience(s) may differ and require different crisis messages depending on their respective relationships to the crisis.

During a crisis, organizations need to follow the steps in the STREMII model to ensure responsive engagement and effective online responses, in real time, and continually monitor, evaluate, and engage in ongoing listening to ensure appropriate and effective response. After a crisis occurs, the model and process do not stop; as organizations must continue to engage with their audience(s), practice social listening for feedback, and implement any necessary changes in order to improve communication when the next crisis happens by adjusting messages and strategies to changing audiences and the nature of the crisis itself.

While the original STREMII model emerged with the context natural disasters like Hurricanes Sandy and Matthew, Stewart \& Young [3] advocated for expanding the model to other types of crises and organizations, agencies and institutions. Moving forward, they desired to survey public relations practitioners and social media strategists to determine the usefulness of the STREMII model and to receive valuable feedback for creating a social listening guide for practitioners.

This current paper will document the methods and procedures we took to survey a broad range of communication and social media professionals about their process for listening socially, their exposure to and understanding of the STREMII model, and their perceptions of whether the model adequately reflects or captures their current practices for listening socially. Lastly, we will discuss what we learned in the process and the next steps for refining the STREMII model and outlining a draft of a practical guide for practitioners.

\section{METHODS OR PROCEDURES}

We aimed to investigate whether or not practitioners in the communications field were following the six steps of the STREMII model during a crisis, with particular attention to the behavior of social listening. We designed a qualitative survey, approved by Ithaca College's Institutional Review Board, to distribute via Qualtrics to public relations practitioners, communication professionals, and social media strategist. In general, we asked for demographic information such as their role or position, age range, industry, type of organization, and the social media and analytic platforms they were currently using. The next section of questions asked participants about social media and communication - in general, such as what type of crises they dealt with, whether their company had a crisis communication plan, with a social media component or strategy, whether they monitor and respond to a crisis on social media and how often, and their description of how they listen socially. The third set of questions corresponded with the STREMII model and socially listening during crises in particular. We uploaded a visual representation of the STREMII model and queried about their familiarity with the model and if the model reflects similar processes they used, and what they would like to see in a practical guide for socially listening.

Based on Young and Stewart's professional connections, we sent out the link to the survey to the Public Relations Society of America internal messaging system and the National Institute for Social Media, and recruited additional participants through other communication and social media professional organizations that do not require a membership as well as groups on LinkedIn. The next section presents a discussion of the results and lessons learned.

\section{RESULTS AND DISCUSSION}

In our search to discover whether practitioners executing social listening in a way that the STREMII model suggests in their practice, we had a total of 62 people engage with this survey. However, as the survey advanced, engagement with certain questions decreased.

Upon asking basic demographics of our respondents, we found that the recurring titles held by our participants include those in higher education, public relations, communications, marketing, and social media specialists. 24 of our respondents experienced a crisis independently or with their organization, the most common being issues of reputation, social media, and death/injury.

22 out of 30 respondents reported to have a crisis plan. Of these respondents, $75 \%$ had a social media component to their plan, while $25 \%$ did not. Additionally, $62 \%$ of these folks have responded to a crisis on social media. This is reflective of the engagement and response part of the STREMII model. Our results show that a large majority are not aware of the STREMII model.

As for the accuracy of the model, 17 respondents reported that the 6 steps of model are mostly reflective of their practice and communication plan, 6 wrote their communication plan partly reflects the model, and 5 stated their communication plan is not reflective of this model. This tells us that the model is mostly accurate in what practitioners are utilized by practitioners.

However, we discovered that steps one, two, and four of the models were the most likely to be followed. Step one, social listening on media, is the most commonly followed. 21 participants agreed that their organization engages in social listening in their practice, while three do not feel that their organization engages with this particular step in the model. Further enhancing the collection of information supporting step 1 in the model, we discovered that a majority, 15 out of 25 , monitored social media for potential crises daily. This figure increases to 20 out of 25 , or $80 \%$, of social media threat 
monitoring when conducted at least 2-3 times per week. This shows us that practitioners, for the most part, are continuously engaging in the living, breathing maintenance and listening agents of their social media channels.

These results show us what practitioners are doing, but the inclusion of case studies in the future can outline whether this is beneficial or not towards navigating a crisis on social media. We have begun the early steps of identifying that this model is applicable in the public relations realms among different sectors of professions. Most notably, the pre-crisis components of the STREMII model, social listening and target audiences, coupled with ongoing evaluation and listening prove to be recognizable behaviors among the surveyed individuals within their existing crisis management practices online. With this in mind, there is a potential opportunity to inform and educate practitioners regarding the value of integrating the other STREMII steps within their crisis management on social media to promote a fluid, effective result for their online stakeholders.

\section{CONCLUSION}

In closing, we have collected data to investigate whether or not practitioners in the communications field were implementing the STREMII model in their practice. We have discovered a baseline to lead us to believe that 1) Most people are not aware of the STREMII model and 2) In most cases; practitioners are following several phases of the STREMII model. Based on suggestions from participants, they wanted the practical guide to include relevant case studies and practical examples with step-by step instructions for implementing STREMII processes. This feedback invites us to continue developing both pedagogical and practical approaches to our STREMII research agenda, and set forth to provide more value and information regarding the implementation of STREMII to practitioners who may find the process meaningful to their work.

Moving forward, there are opportunities to identify case studies, advance qualitative research, and interviews to formulate a systematic guide for social listening using the STREMII model. As crises become increasingly present on social media, and these tools are increasingly popular to communicate with online constituents, the development of a guide to navigate people and organizations through a crisis would be useful for practitioners to improve communication during potential crises. Our next step would be to identify appropriate case studies and guides that would assist practitioners and further promote the relevancy, attainability, and overall benefit to using the STREMII model in practice for crisis management on social media.

\section{Author Biography}

Cory Young (youngc@ithaca.edu, Ph.D., Bowling Green State University, 2001) is an Associate Professor of Communication Management and Design, in the Department of Strategic Communication at Ithaca College in upstate New York.

Hunter Simmons (mhsimmons@ithaca.edu) is a $1^{\text {st }}$ year student at Ithaca College in upstate New York pursuing a Bachelor of Science degree in Communication Management and Design. Class of 2022.

Margaret C. Stewart (m.c.stewart@unf.edu, Ph.D., Indiana University of Pennsylvania, 2013) is an Assistant Professor of Communication Studies in the Department of Communication at University of North Florida in Jacksonville, Florida.

\section{REFERENCES}

[1] Stewart, M.C. \& Wilson, G. B. (2015). The dynamic role of social media during Hurricane \# Sandy: An introduction of the STREMII model to weather the storm of the crisis lifecycle. Computers in Human Behavior, 54, 639-646. https://doi.org/10.1016/j.chb.2015.07.009

[2] Stewart, M. C. \& Young, C. (2018). Revisiting STREMII: Social media crisis communication during Hurricane Matthew. Journal of International Crisis and Risk Communication Research, 1, 279-302. https://doi.org/10.30658/jicrcr.1.2.5

[3] Stewart, M. C., \& Atilano, M. (2018). Reputational threats online: Social media as a simultaneous agent of crisis and tool for response and resolution in the case study of an American academic library. Proceedings of the International Crisis and Risk Communication Conference (pp. 24-26). Orlando, FL. USA. Nicholson School of Communication. https://doi.org/10.30658/icrcc.2018.7 